\title{
BASIC OBSERVATIONAL CHARACTERISTICS OF IMPULSIVE SXR BRIGHTENINGS
}

\author{
M. Tomczak ${ }^{1}$, and T. Mrozek ${ }^{1}$ \\ ${ }^{1}$ Astronomical Institute, Wroctaw University, Kopernika 11, PL-51-622 Wroctaw, Poland
}

\begin{abstract}
25 flares showing impulsive SXR brightenings have been investigated. The Yohkoh/SXT images have been used. The individual regions of the SXR response for each flare have been carefully localized. Some observational characteristics of them have been obtained. The results are presented in the form of histograms. For more comprehensive analysis HXR data from the Yohkoh/HXT has been incorporated. For several cases the velocity of plasma motions due to the chromospheric evaporation has been estimated directly from the SXR images. The obtained results will help to distinguish impulsive SXR brightenings from other effects and will be useful for theoretical modeling of the flare impulsive phase. $\odot 2002$ COSPAR. Published by Elsevier Science Ltd. All rights reserved.
\end{abstract}

\section{INTRODUCTION}

A very good quality of images derived from the Soft X-ray Telescope (SXT) aboard the Japanese satellite Yohkoh allows to separate a short-time, rapidly-varying component in soft X-rays (SXR) of solar flares. The impulsive soft $\mathrm{X}$-ray brightenings are seen during the impulsive phase of many flares. Time interval of their occurrence as well as location at footpoints of the flaring magnetic structure strongly suggest that impulsive SXR brightenings are due to precipitation of non-thermal electron beams (Strong et al. 1994). Hudson et al. (1994) showed that impulsive SXR brightenings are caused by thermal emission of plasma heated by non-thermal electrons and evaporated from the deeper atmosphere into the flaring structure. Tomczak (1999) argued that impulsive SXR brightenings are produced mainly by low-energy non-thermal electrons.

Regarding the above relationships, results of detailed investigation of the impulsive SXR brightenings offer additional information about the non-thermal electron precipitation and the chromospheric evaporation. For example, the velocities of the chromospheric evaporation have been estimated directly from the SXT images (Tomczak 1997a, 1997b). Moreover, it has been showed that impulsive SXR brightenings are responsible for the Neupert effect (Tomczak 1999).

The previously performed research dedicated to the impulsive SXR brightenings was concentrated on a detailed analysis of individual selected flares. On that basis it is difficult to extract typical characteristics of impulsive SXR brightenings. The knowledge of them seems to be very important for the right analysis of some complex events e.g. those analyzed by Harra-Murnion et al. (1997) for which two different explanations were presented: the extra-strong impulsive SXR brightening in the footpoint of a single loop or an additional energy release in a small loop situated there. Our purpose was to collect as many as possible observations of the impulsive SXR brightenings to obtain statistically important limits for the values of parameters describing this phenomenon. Preliminary results are presented in this paper.

\section{ANALYSIS}

Table 1 presents a list of 25 flares that have been analyzed. Their impulsive phases were well-observed by Yohkoh and their impulsive SXR brightenings were visible after a prompt inspection of the observations. The SXT images of each event were reduced with the standard SXT PREP routine (Morrison 1994). To separate areas of impulsive SXR brightenings from other parts of the flaring structure the light curves of individual pixels $(2.45 \operatorname{arcsec} \times 2.45 \mathrm{arcsec})$ have been carefully analyzed. We used the SXT images made 
Table 1. List of investigated flares ${ }^{a}$

\begin{tabular}{|c|c|c|c|c|c|c|c|c|c|c|}
\hline (1) & (2) & (3) & (4) & (5) & (6) & $(7)$ & $(8)$ & (9) & $(10)$ & $(11)$ \\
\hline 1 & 2-NOV-91 & $06: 47$ & $06: 53$ & M9.0 & $1 \mathrm{~B}$ & S13 W61 & 6891 & 4 & 5 & $130-150$ \\
\hline 2 & 9-NOV-91 & 03:13 & 03:20 & M1.5 & SN & N20 E08 & 6918 & 2 & 2 & \\
\hline 3 & 10-NOV-91 & $20: 10$ & $20: 13$ & M7.9 & $1 \mathrm{~N}$ & S15 E43 & 6919 & 3 & 3 & \\
\hline 4 & 19-NOV-91 & $09: 29$ & 09:32 & C8.5 & $1 F$ & S12 W60 & 6919 & 5 & 5 & $280-560$ \\
\hline 5 & 13-JAN-92 & $17: 29$ & $17: 34$ & M2.0 & - & S15 Wlimb & 6994 & 2 & 2 & $260-310$ \\
\hline 6 & 26-JAN-92 & $15: 28$ & $15: 33$ & $\mathrm{X} 1.0$ & 3B & S16 W66 & 7012 & 6 & 7 & $360-640$ \\
\hline 7 & 14-FEB-92 & 23:07 & 23:10 & M7.0 & $2 \mathrm{~B}$ & S13 E02 & 7056 & 4 & 4 & $220-380$ \\
\hline 8 & 17-FEB-92 & $15: 42$ & $15: 46$ & M1.9 & SF & N16 W81 & 7050 & 3 & 5 & \\
\hline 9 & 28-JUN-92 & $13: 57$ & $14: 24$ & M1.6 & - & N15 Elimb & 7216 & 7 & 14 & $400-720$ \\
\hline 10 & 8-JUL-92 & $09: 48$ & 09:50 & $\mathrm{X} 1.2$ & $1 B$ & S11 E46 & 7220 & 4 & 8 & $280-450$ \\
\hline 11 & 15-JUL-92 & 02:00 & 02:03 & C4.2 & - & S11 W36 & 7222 & 4 & 6 & \\
\hline 12 & 11-AUG-92 & 13:48 & $13: 51$ & C7.2 & $1 \mathrm{~N}$ & S11 W45 & 7248 & 3 & 3 & \\
\hline 13 & 11-AUG-92 & $22: 25$ & $22: 28$ & M1.4 & - & N15 Elimb & 7260 & 5 & 5 & $380-560$ \\
\hline 14 & 6-SEP-92 & 05:16 & $05: 19$ & M2.4 & $2 \mathrm{~N}$ & S09 W39 & 7270 & 3 & 5 & \\
\hline 15 & 6-SEP-92 & 09:04 & 09:07 & M3.3 & $1 \mathrm{~N}$ & S11 W38 & 7270 & 3 & 3 & \\
\hline 16 & 11-SEP-92 & 03:00 & 03:02 & M1.0 & - & N17 E40 & 7276 & 3 & 5 & \\
\hline 17 & 12-SEP-92 & $15: 39$ & $15: 47$ & C5.2 & SN & N18 E21 & 7276 & 2 & 2 & \\
\hline 18 & 2-FEB-93 & 03:46 & 03:49 & C6.3 & $1 \mathrm{~N}$ & S16 E13 & 7412 & 3 & 4 & \\
\hline 19 & 3-FEB-93 & 14:59 & 15:04 & C8.8 & $1 F$ & N10 E64 & 7417 & 2 & 2 & \\
\hline 20 & 6-FEB-93 & $20: 47$ & 20:50 & C7.5 & SF & S08 E54 & 7420 & 2 & 2 & \\
\hline 21 & 2-MAR-93 & $15: 05$ & $15: 10$ & C5.0 & SF & S07 E82 & 7440 & 2 & 2 & 240 \\
\hline 22 & 27-SEP-93 & $10: 52$ & $10: 54$ & C5.7 & SF & N11 E80 & 7590 & 3 & 5 & \\
\hline 23 & $30-N O V-93$ & 06:04 & 06:08 & C9.2 & - & S20 Elimb & 7627 & 5 & 5 & $580-630$ \\
\hline 24 & 16-JAN-94 & 23:20 & 23:25 & M6.1 & $1 B$ & N09 E73 & 7654 & 3 & 5 & \\
\hline 25 & 30-JUN-94 & 21:21 & $21: 24$ & M2.5 & 1B & S12 E27 & 7742 & 2 & 3 & \\
\hline
\end{tabular}

a (1) - number of event; (2) - date; (3) - HXR maximum time [UT]; (4) GOES maximum time [UT]; (5) - GOES class; (6) - H $\alpha$ importance; (7) - location; (8) - NOAA AR; (9) - number of regions of the SXR response; (10) - number of the impulsive SXR brightening; (11) - range of evaporation velocities $\left[\mathrm{km} \mathrm{s}^{-1}\right]$.

with the $11.6 \mu \mathrm{m}$ Al filter (Al12) in which the impulsive SXR brightenings are most evident. Temporal resolution of the investigated light curves was typically 8-14 s. An exception are events Nos. 18-20 for which the images made with the $119 \mu \mathrm{m}$ beryllium filter (Be119) were available with 2-s. time resolution.

Only the pixels which reached the statistically important maximum of brightness during the impulsive phase were taken into consideration. It has been found that these pixels were concentrated in several (2-7) regions which can be localized at the footpoints of a flaring structure. Every region is called the region of the SXR response, i.e. showing impulsive SXR brightenings. Usually hard X-ray (HXR) emission sources were also seen in these regions, looking at the images obtained by the Yohkoh Hard X-ray Telescope (HXT). An example of the relative position of the regions of the SXR response and of the HXR emission sources is presented in Fig. 1.

For further analysis the SXT signal was accumulated over each region of the SXR response. The light curves obtained in this way contain a long-time, slowly-varying component which is caused by other factors than non-thermal electrons (e.g. thermal conduction, instrumental factors). Therefore, a net signal describing a pure SXR response due to the non-thermal electrons has been estimated by a background subtraction. It has been assumed that the contribution of the factors other than non-thermal electrons to the SXR radiation of the footpoints was completely outside the impulsive phase, and rose linearly in time during the impulsive phase (Tomczak 1999). Examples of both types of light curve, i.e. the total before the background subtraction and the net, as well as the HXR light curve, are presented in Fig. 2. 


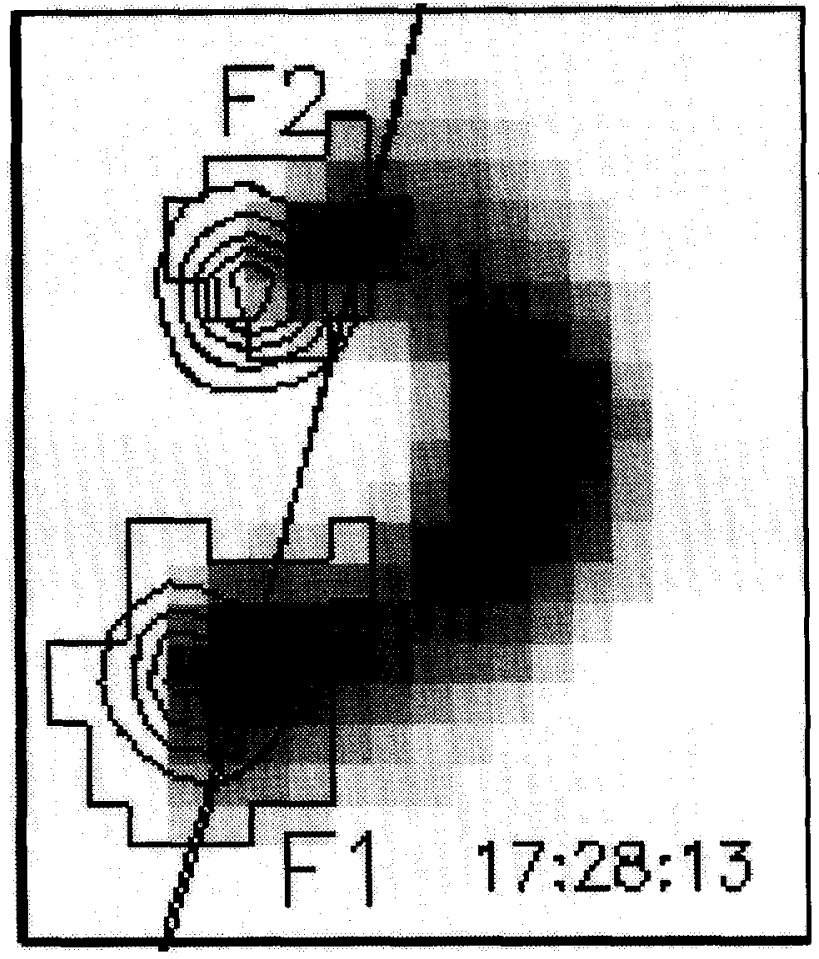

Fig. 1. The SXT/Be119 image of 13 January 1992 flare (event No. 5) obtained at the maximum of the HXR light curve. The pixel size is 2.45 arcsec $\times 2.45$ arcsec. The regions of the SXR response, $F 1$ and $F 2$, are marked. Contours of the HXT/(channel M2) emission are overplotted $(90,70,50,30$, and $10 \%$ of the maximum intensity). Solar north is up, east - to the left. The solar limb is marked by the straight line.

The net light curves have been used to obtain following characteristics of the impulsive SXR brightenings: (1) FWHM duration time $t-a$ time interval in which the signal was above the half of the maximum value; (2) time profile characteristics measured by the ratios: $t_{r} / t$, and $t_{d} / t$, where $t_{r}$, and $t_{d}$ are the time intervals in which the signal was above the half of the maximum value, during the phases of rise and decay, respectively; (3) relative brightness $I_{F} / I_{\text {tot }}-$ a part of the whole SXR emission of the flare which was due to the non-thermal electron heating in a considered footpoint; (4) SXR/HXR delay - a time difference between the maxima of the net SXR light curve and the HXR light curve; (5) relative duration of the response due to the non-thermal electrons in SXR and HXR radiation $t / t_{H X R}$, where $t_{H X R}$ is the FWHM duration in $H X R$ radiation.
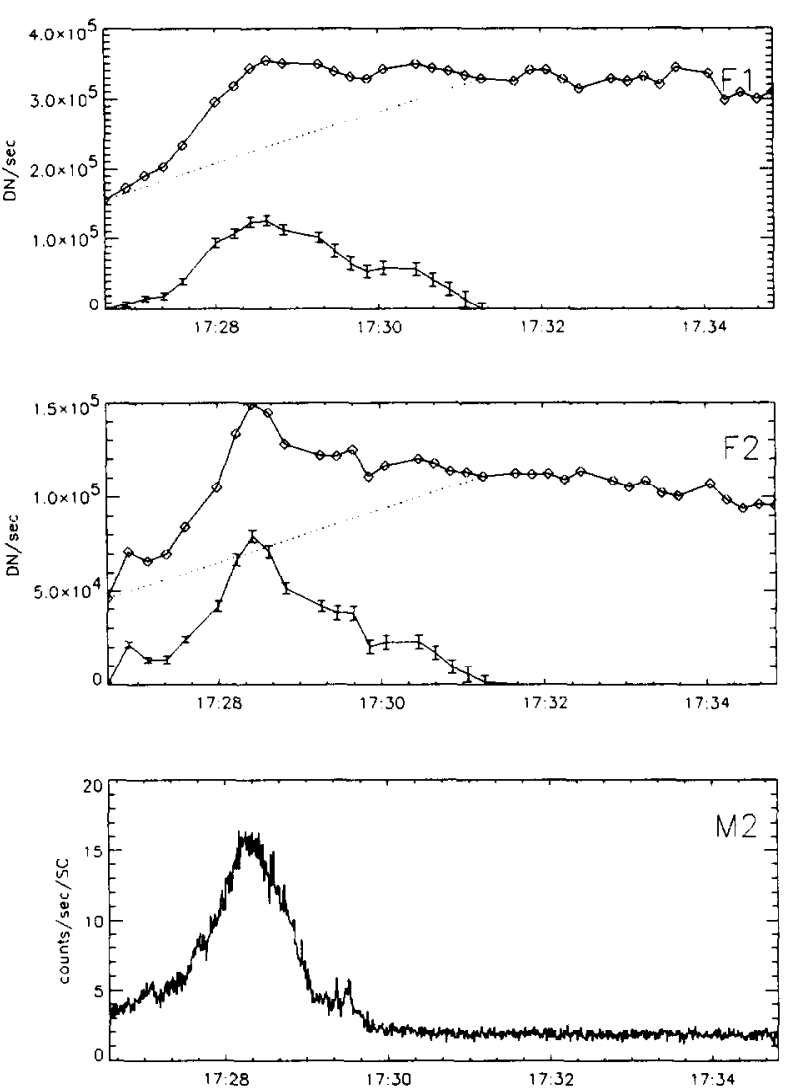

Fig. 2. The SXT/Al12 light curves of regions of the SXR response, $F 1$ and $F 2$, marked in Fig. 1. The total as well as the net light curves are presented. The subtracted background is marked by the dotted line. Moreover, the total HXT/M2 light curve is showed. See text for further details.

For the maximum of the net light curves the temperatures and the emission measures have been estimated from the signal ratio in the Be119 and the Al12 images according to the filter ratio method (Hara et al. 1992). Values of the above parameters were averaged over the regions of the SXR response.

Actual sizes of the regions of the SXR response have been spread due to the Point Spread Function of the SXT (Martens et al. 1995). To eliminate somewhat this effect their FWHM sizes have been considered i.e. only those pixels were included for which the signal was above a half of the brightest one at the maximum of the light curve. 

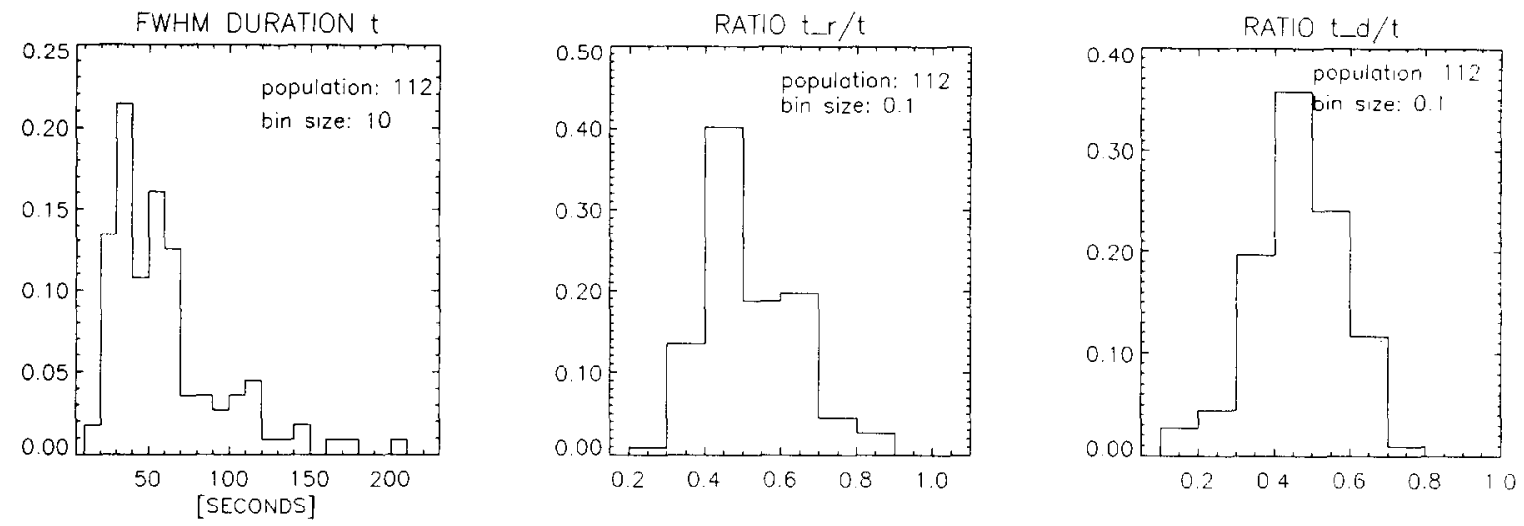

Fig. 3. Normalized histograms of the FWHM duration $t$, the ratio $t_{r} / t$, and the ratio $t_{d} / t$ (left, middle, and right panel, respectively) for investigated impulsive SXR brightenings. See text for further details.
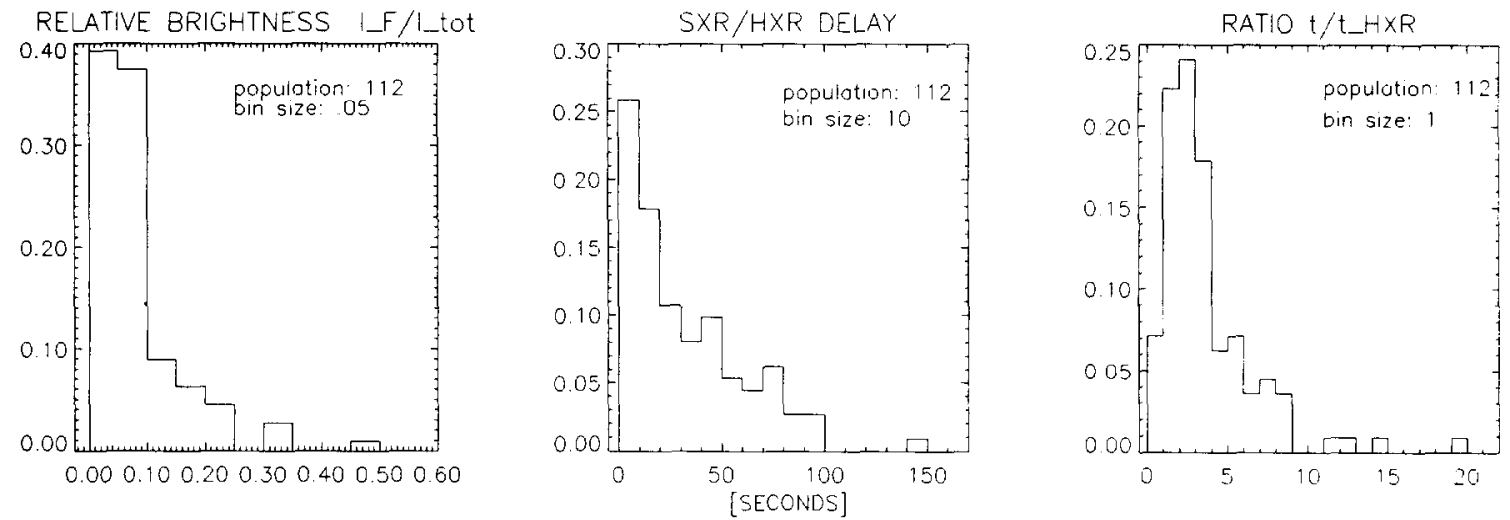

Fig. 4. Normalized histograms of the ratio $I_{F} / I_{t o t}$, the SXR/HXR delay, and the ratio $t / t_{H X R}$ (left, middle, and right panel, respectively) for investigated impulsive SXR brightenings. See text for further details.

\section{RESULTS}

In summary, 85 regions showing 112 impulsive SXR brightenings have been found for the investigated flares. For each of them the observational characteristics defined in the previous Section have been obtained. Results are presented in the form of normalized histograms in Figs. 3-5. Each histogram contains information about number of events and bin size. The bin size of each histogram is taken to be equal to uncertainties of the values distribution of which is shown by the histogram.

In the histogram of FWHM duration (Fig. 3, left panel) about $75 \%$ of the population is concentrated within the interval of 20-70 s. Longer durations are typical for the events in which the impulsive phase has a complex appearance showing several separated HXR bursts. Therefore, we expect that such cases are due to an overlap of the SXR responses to several non-thermal electron beams.

Time profile of impulsive SXR brightenings is almost symmetrical. In the histograms showing the ratios $t_{r} / t$ and $t_{d} / t$ (Fig. 3, middle and right panel, respectively) above $90 \%$ of the population is within the interval of $0.3-0.7$. The values outside this interval are due to complex light curves in which the individual responses cannot be resolved.

Brightness of individual region of the SXR response is distinctly weaker than SXR emission of the whole flaring structure - above $75 \%$ of the population in the histogram showing $I_{F} / I_{\text {tot }}$ (Fig. 4, left panel) falls within two lowest bins i.e. below 0.1. This result allows to doubt about the interpretation of the 27 September 1993 flare considering it to be an extra-strong impulsive SXR brightening (Harra-Murnion et al. 1997). However, we should remember that very often impulsive SXR brightenings occur in several regions simultaneously, so the total SXR response due to non-thermal electrons reaches $20-30 \%$ of the whole SXR 

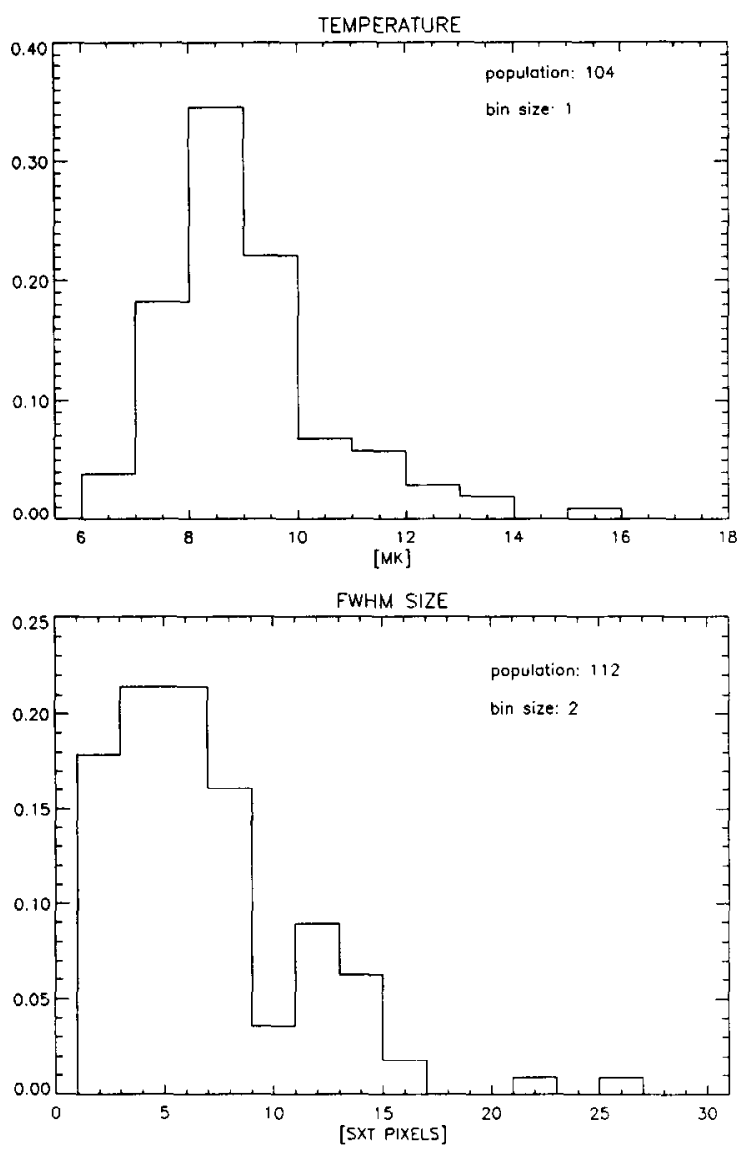

Fig 5. Normalized histograms of the temperature, and the FWHM size (top, and bottom panel, respectively) for investigated regions of the SXR response. See text for further details.

emission of the flare.

For about $45 \%$ of the population the delay of the maximum of SXR net light curve in comparison with the HXR light-curve peak is actually below the temporal resolution of the SXT (Fig. 4, middle panel). It means that for the events from this group the response of the chromosphere to non-thermal electrons was almost immediate. On the other hand, for about $55 \%$ of events the delay was evidently larger, up to $\sim 10^{2}$ s. Excluding some complex events, we can conclude that quite often an inertia of the chromosphere in its response to non-thermal electrons is seen. We expect that the differences in the delay can be due to the differences in the energy spectrum of non-thermal electrons.

The second characteristic which shows the inertia of the chromospheric response to non-thermal electrons is the ratio $t / t_{H X R}$ of FWHM duration of the impulsive SXR brightening, $t$, and of the HXR burst,
$t_{H X R}$. The histogram made for this ratio (Fig. 4, right panel) has the maximum within the interval 23. For about $65 \%$ of the population the impulsive SXR brightening lasts up to 4 times longer than the HXR burst.

Temperatures obtained for regions of the SXR response are collected in Fig. 5 (top panel). For about $75 \%$ of events the values are within the interval of 7$10 \mathrm{MK}$. Events with the temperatures above $10 \mathrm{MK}$ in the histogram correspond to a geometrical configuration in which the footpoint and the bright loop-top kernel were situated nearly along the line-of-sight.

The FWHM sizes of regions of the SXR response are typically rather small - above $75 \%$ of the population is concentrated within the interval of 1-9 SXT pixels (see Fig. 5, bottom panel). This result can suggest that actual sizes of the non-thermal electron beams are below the spatial resolution of the SXT. Events with larger sizes probably represent the cases when some non-thermal electron beams have not been resolved.

For some regions of the SXR response a systematic trend has been found. Namely, the mornent of the impulsive SXR brightening maximum depended on the situation in the flaring structurc - light curves of the pixels located higher above the limb showed their maximum later in time. We consider this effect as a manifestation of the chromospheric evaporation. Velocities of the plasma upflow have been obtained from plots: (time of maximum)-versus-(position along the structure). The results are presented in the last column of Table 1.

\section{CONCLUSIONS}

On the basis of the histograms presented in Figs. 35 we can describe properties of something like a "typical" impulsive SXR brightening. It lasts about 0.5$1 \mathrm{~min}$. and has a quasi-symmetrical time profile. The FWHM diameter of the region of the SXR response is about 2-8 arcsec. Its temperature is below $10 \mathrm{MK}$. The pixels showing the impulsive SXR brightening are often the brightest in the flaring structure during the impulsive phase, however their total contribution to the SXR emission of the whole structure is only rarely above $30 \%$. In comparison with the HXR burst the impulsive SXR brightening shows a delay and lasts several times longer.

The described characteristics should be helpful to distinguish the impulsive SXR brightening from other effects. Particularly, any event resembling the impulsive SXR brightening for which observational characteristics are evidently different from those described 
in the previous paragraph should be interpreted with a special caution. The collected characteristics should be useful also for theoretical modeling of the flare impulsive phase.

A generally good spatial and temporal correlation between impulsive SXR brightenings and HXR bursts strongly suggests the same origin - non-thermal electron beams. For this reason the SXT images signifcantly complements the HXT images in the monitoring of the non-thermal electron precipitation during the flare impulsive phase. The role of the SXT in such a investigation is so important because it offers better spatial resolution and dynamical range than the HXT.

The measurement of velocities of plasma motions due to the chromospheric evaporation directly from the SXT images is the additional method of the investigation of such an effect. Especially important is that the developed method complements the classical - blueshifts in the SXR spectra - for the flares occurred close to the solar limb. The obtained range of values, $150-700 \mathrm{~km} / \mathrm{s}$, is similar to those estimated from the spectral measurements (e.g. Bentley et al. 1994).

Often the flare evolution during the impulsive phase is very complicated - we observed a response to nonthermal electrons in several footpoints simultaneously. Moreover, different footpoints reacted in different moments of time. Very convenient in the physical interpretation of such complex events is the turbulent kernel model (Jakimiec et al. 1998). In this model the energy of flares is released in many current sheets inside a region where the magnetic field lines are tangled. Each reconnection with an external line of force opens temporarily the kernel and allows to escape some non-thermal electrons outside the kernel. If the external lines are rooted somewhere in the chromosphere we observe there the HXR and SXR response.

A more comprehensive list of flares including a quantitative HXR analysis is in progress.

\section{ACKNOWLEDGMENTS}

The Yohkoh satellite is a project of the Institute of Space and Astronautical Science of Japan. We thank Prof. J. Jakimiec for many useful comments and discussions. This work was supported by the KBN grant No. 2 P03D 01614.

\section{REFERENCES}

Bentley, R.D., G.A. Doschek, G.M. Simnett, M.L. Rilee, J.T. Mariska, J.L Culhane, T. Kosugi, and T. Watanabe, The Correlation of Solar Flare Hard X-ray Bursts with Doppler Blueshifted Soft X-ray Flare Emission, Astrophysical J. Letters, 421, L55-L58, 1994.

Hara, H., S. Tsuneta, J.R. Lemen, L.W. Acton, and J.M. McTiernan, High-Temperature Plasma in Active Regions Observed with the Soft X-ray Telescope Aboard Yohkoh, Publ. Astron. Soc. Japan, 44, L135L140, 1992.

Harra-Murnion, L.K., J.L. Culhane, H.S. Hudson, T. Fujiwara, T. Kato, and A.C. Sterling, Isolating the Footpoints Characteristics of a Solar Flare Loop, Solar Phys., 171, 103-122, 1997.

Hudson, H., K. Strong, B. Dennis, D. Zarro, M. Inda, T. Kosugi, and T. Sakao, Impulsive Behavior in Solar X-Radiation, Astrophysical J. Letters, 422, L25-L27, 1994.

Jakimiec, J., M. Tomczak, R. Falewicz, K.J.H. Phillips, and A. Fludra, The Bright Loop-Top Kernels in Yohkoh X-ray Flares, Astron. Astrophys., 334, 1112-1122, 1998.

Martens, P.C., L.W. Acton, and J.R. Lemen, The Point Spread Function of the Soft X-ray Telescope Aboard Yohkoh, Solar Phys., 157, 141-168, 1995.

Morrison, M. Yohkoh Analysis Guide, Lockheed Palo Alto Res. Lab. Rep. LMCSC-P098510, 1994.

Strong, K., H. Hudson, and B. Dennis, Evidence for Impulsive Soft X-ray Bursts during Flares, X-Ray Solar Physics from Yohkoh, (eds.) Y. Uchida, T. Watanabe, K. Shibata, and H.S. Hudson, Universal Academy Press (Tokyo), pp. 65-69, 1994.

Tomczak, M., The Impulsive Phase of the Arcade Flare of 28 June 1992, 14:24 UT, Astron. Astrophys., 317, 223-231, 1997a.

Tomczak, M., Investigation of Impulsive Soft X-ray Brightening in Solar Flares, Adv. Space Res., 20(12), 2323-2326, $1997 \mathrm{~b}$.

Tomczak, M., Yohkoh Observations of the Neupert Effect, Astron. Astrophys., 342, 583-591, 1999. 